\title{
Does Vitamin D Deficiency is a Risk Factor for Diabetic Nephropathy in Egyptian Diabetic Patients?
}

\author{
Mohamed A Helaly $^{1 *}$, Amr El-Baz ${ }^{1}$ and Nancy Mahsoub ${ }^{2}$ \\ ${ }^{1}$ Departements of Internal Medicine, Egypt \\ ${ }^{2}$ Clinical Pathology, Specialized Medical Hospital, Faculty of Medicine, Mansoura University, Egypt \\ *Corresponding author: Mohamed A Helaly, Specialized Medical Hospital, Al-Gomhoria Street, Mansoura Egypt
}

\section{ARTICLE INFO}

Received: 幽 February 08, 2020

Published: 幽 February 18, 2020

Citation: Mohamed A $\mathrm{H}^{*}$, Amr E and Nancy M. Does Vitamin D Deficiency is a Risk Factor for Diabetic Nephropathy in Egyptian Diabetic Patients?. Biomed J Sci \& Tech Res 25(4)-2020. BJSTR. MS.ID.004237.

\begin{abstract}
Vitamin D deficiency was found to have higher prevalence in poorly controlled diabetic patients. Also, vitamin D deficiency was found to have higher prevalence in diabetic micro vascular complications. On the other hand, patients with CKD in different stages suffer from vitamin D deficiency. Our study was conducted on 100 diabetic patients (50 diabetic patients without nephropathy and 50 diabetic patients with nephropathy) in addition to 50 healthy subjects as a control group; to evaluate level of vitamin D in both control group, diabetic patients without nephropathy and diabetic patients with nephropathy throughout different stages of chronic kidney disease (CKD).This study revealed that there was a significant difference in the level of vitamin D between control group and diabetic patients group. It tended to be lower in diabetic patients; also, there was highly significant difference between diabetic patients without nephropathy and diabetic patients with nephropathy. Vitamin D level was lower in diabetic patients with nephropathy group; also, there was significant difference between different stages of CKD in diabetic patients with nephropathy group. Level of vitamin D showed a progressive decline throughout different stages of CKD, it was the least in stage 3 CKD. In diabetic patients with nephropathy there were negative correlations between serum creatinine and albumin/creatinine ratio with vitamin D while there was positive correlation between serum albumin and eGFR with vitamin $D$.
\end{abstract}

\section{Introduction}

Type 2 diabetes mellitus is a group of metabolic diseases as result of chronic hyperglycemia resulting from defects in insulin secretion, insulin action, or both [1]. Diabetic nephropathy is leading cause of end stage chronic kidney disease in patients starting renal replacement therapy and affects $40 \%$ of type 1 and type 2 diabetic patients [2]. The natural course of diabetic nephropathy is characterized by specific renal morphological and functional changes. The first renal change occur is micro-albuminuria (30 $300 \mathrm{mg}$ / day), glomerular hyperfiltration, glomerular and renal hypertrophy, increased basement membrane thickness, and mesangial expansion with the accumulation of extracellular matrix proteins such as collagen, fibronectin, and laminin. Advanced diabetic nephropathy is characterized by macroalbuminuria $(>300$ $\mathrm{mg} /$ day) [3]. Vitamin D known as sunshine vitamin is fat soluble vitamin produced after exposure of the skin to sunlight [4]. Vitamin
D from the diet is biologically inactive; activation requires enzymatic conversion (hydroxylation) in the liver and kidney [5]. The renin angiotensin system (RAAS) is a major mediator of progressive renal injury in diabetic nephropathy. Vitamin $D$ negatively regulates the RAAS by suppressing rennin expression and thus plays renoprotective role in diabetic nephropathy. Combination therapy with an RAAS inhibitor and vitamin D analogue markedly ameliorates renal injuries due to blockade of compensatory rennin increase by the analogue [6].

\section{Aim of the Study}

Is to study serum level of vitamin D in Egyptian diabetic patients with and without nephropathy.

\section{Subjects and Methods}

This study was conducted at Mansoura University, Specialized Medical Hospital, (inpatient and outpatient clinic internal medicine 
department).It was approved by the institutional review board, and a written informed consent was obtained from all participants and met the requirements of local ethical committee ; Mansoura University. The study was done on 100 diabetic patients and 50 control subjects and lasting for 12 months (from January 2017 to January 2018). Patients with established diagnosis of diabetes mellitus on basis of medical history, clinical examination and laboratory tests [7]. The study included 100 diabetic patients (90 male and 10 female) between the ages of 18 - 73 years old, while the control group was 50 subjects ( 40 male and 10 female) between the ages of 30 - 64 years old. The diabetic patients group was divided into two main groups' diabetic patients without nephropathy group and diabetic patients with nephropathy group based on ADA diagnostic criteria on diabetic nephropathy which is the persistent presence of elevated urinary albumin excretion (albuminuria),low estimated glomerular filtration rate (eGFR) in the absence of signs or symptoms of other primary causes of kidney damage [8]. The following exclusions criteria were considered in selecting the diabetic patients :

a) Any hypertensive patients not related to diabetic complication

b) Patients with heart diseases, valvular heart lesions, cardiomyopathy or pericardial diseases.

c) Any patient with pre-existing renal disease not related to diabetic complications.

d) Any patient with systemic diseases that affect kidney as Systemic lupus.

e) Patients with severe liver diseases or hepatorenal syndrome.

\section{Result}

Table 1: Demographic criteria, of studied subjects.

\begin{tabular}{|c|c|c|c|}
\hline \multicolumn{2}{|c|}{ Diabetic Patients (100) } & Control group (50) & $\mathbf{P}$ \\
\hline \multicolumn{4}{|c|}{ Age } \\
\hline Mean \pm SD & $48.61 \pm 13.01$ & $46.92 \pm 6.8$ & \multirow{2}{*}{0.298} \\
\hline Range & $18-73$ & $30-64$ & \\
\hline \multicolumn{4}{|c|}{ Gender } \\
\hline Female & $10(10)$ & $10(20)$ & \multirow{2}{*}{0.056} \\
\hline Male & $90(90)$ & $40(80)$ & \\
\hline \multicolumn{4}{|c|}{ BMI } \\
\hline Mean \pm SD & $28.8 \pm 3.56$ & $26.69 \pm 3.69$ & \multirow{2}{*}{$0.001^{* *}$} \\
\hline Range & $21.79-40.23$ & $19.11-32.32$ & \\
\hline \multicolumn{4}{|c|}{ Systolic Blood Pressure } \\
\hline Mean \pm SD & $120.5 \pm 8.18$ & $115.9 \pm 9.13$ & \multirow{2}{*}{$0.002^{*}$} \\
\hline Range & $100-140$ & $100-130$ & \\
\hline
\end{tabular}

f) Patients on hemodialysis and patients with GFR $<$ I5 ml/

\section{Patients Included in this Study Were Subjected To}

Thorough history taking and clinical assessment including type of diabetic medications currently used, history of diabetic coma during last year, history of diabetic complications already confirmed, calculation of BMI by weight/height2(weight in $\mathrm{kg}$, height in $\mathrm{m}$ ), calculation of estimated glomerular filtration rate (eGFR) calculated by Cockcroft-Gault equation eGFR for males = (I40-age) X wt. (kg) / (72x serum creatinine) eGFR for females = (I40-age) X wt. (kg) / (72x serum creatinine) x 0.8 [9].

\section{Laboratory Assessment}

The following laboratory tests were done in order to evaluate the patients as serum creatinine, serum albumin, glycosylated hemoglobin (HbA1c), urinary albumin/creatinine ratio, serum $25(\mathrm{OH})$ vitamin D level. The laboratory tests were done using Enzyme-Linked Immunosorbent Assay (ELISA) test which was tested in human plasma and serum samples. The diabetic patient with nephropathy was subdivided according to their eGFR to five groups:

a) CKD stage 1: more than $90 \mathrm{ml} / \mathrm{min} / \mathrm{I} .73 \mathrm{~m} 2$.

b) CKD stage $2: 89-60 \mathrm{ml} / \mathrm{min} / \mathrm{I} .73 \mathrm{~m} 2$.

c) CKD stage $3: 59-30 \mathrm{ml} / \mathrm{min} / \mathrm{I} .73 \mathrm{~m} 2$.

d) CKD stage $4: 29-15 \mathrm{ml} / \mathrm{min} / \mathrm{I} .73 \mathrm{~m} 2$.

e) CKD stage 5 : less than $15 \mathrm{ml} / \mathrm{min} / \mathrm{I} .73 \mathrm{~m} 2$.

f) CKD stages $4 \& 5$ were excluded in our study. $\min / \mathrm{I} .73 \mathrm{~m} 2$. 


\begin{tabular}{|c|c|c|c|}
\hline Mean \pm SD & $79.7 \pm 7.31$ & $74.7 \pm 8.77$ & $<0.001^{* *}$ \\
\cline { 1 - 3 } & $65-90$ & $60-90$ & \\
\hline
\end{tabular}

Note: $\mathrm{p} \leq 0.00 \mathrm{I}$ is highly significant; BMI: Body Mas

s Index.

Table 1 showed that there were non-significant differences between diabetic patients and control group regarding age and gender. There were significant differences between diabetic patients and control group regarding BMI, systolic blood pressure and diastolic blood pressure. They were higher in diabetic group. Table 2 showed that in diabetic group, serum creatinine was higher, but serum vitamin D, albumin, eGFR were lower than control group. Table 3 showed that there were non-significant differences between diabetic patients with nephropathy and those without nephropathy regarding age and gender. Systolic blood pressure was higher in diabetic group with nephropathy. Table 4 showed that vitamin D levels and eGFR were lower in diabetic group with nephropathy than those without it. However, serum creatinine, albumin /creatinine ratio and HbA1c, were higher in diabetic group with nephropathy. Table 5 showed that serum level of vitamin D was lower in grade 3 nephropathy than grade $1 \& 2$. Serum creatinine and albumin $\backslash$ creatinine ratio were higher in grade 3 nephropathy than other grades. However, serum albumin and eGFR were lower in them. Table 6 showed that Vitamin D level had a significant positive correlation with eGFR and a negative correlation with serum creatinine in control group. In diabetic patients without nephropathy, vitamin D had a significant positive correlation with serum albumin and negative one with HbAIc. In diabetic patients with nephropathy, there were significant negative correlations of serum level of vitamin D with creatinine, albumin/creatinine ratio and $\mathrm{HbA1c}$. But it had a significant positive correlation with serum albumin and eGFR in the same group.

Table 2: laboratory investigations of studied groups.

\begin{tabular}{|c|c|c|c|}
\hline Diabetic Patients(100) & & Control Group (50) & $\mathrm{P}$ \\
\hline \multicolumn{4}{|c|}{ Vitamin D } \\
\hline Mean \pm SD & $7.02 \pm 5.73$ & $12.14 \pm 6.1$ & \multirow{2}{*}{$<0.001^{* *}$} \\
\hline Range (ng/ml) & $1.7-38$ & $4.4-29.1$ & \\
\hline \multicolumn{4}{|l|}{ Serum Creatinine } \\
\hline Mean \pm SD & $1.24 \pm 0.38$ & $0.95 \pm 0.21$ & \multirow{2}{*}{$<0.001^{* *}$} \\
\hline Range (mg/dl) & $0.6-2.5$ & $0.5-1.4$ & \\
\hline \multicolumn{4}{|c|}{ Serum Albumin } \\
\hline Mean \pm SD & $3.8 \pm 0.33$ & $4.04 \pm 0.27$ & \multirow{2}{*}{$<0.001^{* *}$} \\
\hline Range $(g \backslash d l)$ & $2.8-4.8$ & $3.5-4.8$ & \\
\hline \multicolumn{4}{|c|}{ eGFR: } \\
\hline Mean \pm SD & $93.93 \pm 20.29$ & $114.42 \pm 14.22$ & \multirow{2}{*}{$<0.001^{* *}$} \\
\hline Range (ml/min/ I.73 m2) & $41.0-126.13$ & $87.3-146.4 \mathrm{I}$ & \\
\hline
\end{tabular}

Table 3: Comparison between groups with and without nephropathy.

\begin{tabular}{|c|c|c|c|}
\hline \multicolumn{2}{|c|}{ Diabetics Without Nephropathy (50) } & Diabetics with Nephropathy (50) & $\mathbf{P}$ \\
\hline \multicolumn{4}{|c|}{ Age } \\
\hline Mean \pm SD & $47.48 \pm 12.91$ & $49.74 \pm 13.15$ & \multirow{2}{*}{0.388} \\
\hline Range & $18-73$ & $20-71$ & \\
\hline \multicolumn{4}{|c|}{ Gender } \\
\hline Female & $5(10)$ & $4(8)$ & \multirow{2}{*}{ I } \\
\hline Male & $45(90)$ & $46(92)$ & \\
\hline \multicolumn{4}{|c|}{ BMI } \\
\hline Mean \pm SD & $28.85 \pm 4.23$ & $28.75 \pm 2.78$ & \multirow{2}{*}{0.888} \\
\hline Range & $21.79-40.23$ & $21.88-35.56$ & \\
\hline \multicolumn{4}{|c|}{ Systolic Blood Pressure } \\
\hline Mean \pm SD & $117.8 \pm 7.57$ & $123.2 \pm 7.94$ & \multirow{2}{*}{$0.001^{* *}$} \\
\hline Range & $100-130$ & $110-140$ & \\
\hline
\end{tabular}




\begin{tabular}{|c|c|c|c|}
\hline \multicolumn{3}{|c|}{ Diastolic Blood Pressure } \\
\hline Mean \pm SD & $78.7 \pm 7.61$ & $80.7 \pm 6.93$ & 0.173 \\
\hline Range & $65-90$ & $70-90$ & \multirow{2}{*}{0} \\
\hline
\end{tabular}

Table 4: Laboratory investigations of diabetic patients with and without nephropathy.

\begin{tabular}{|c|c|c|c|}
\hline \multicolumn{2}{|c|}{ Diabetics Without Nephropathy (50) } & Diabetics with Nephropathy (50) & $\mathbf{P}$ \\
\hline \multicolumn{4}{|c|}{ Age } \\
\hline Mean \pm SD & $47.48 \pm 12.91$ & $49.74 \pm 13.15$ & \multirow{2}{*}{0.388} \\
\hline Range & $18-73$ & $20-71$ & \\
\hline \multicolumn{4}{|c|}{ Gender } \\
\hline Female & $5(10)$ & $4(8)$ & \multirow{2}{*}{ I } \\
\hline Male & $45(90)$ & $46(92)$ & \\
\hline \multicolumn{4}{|c|}{ BMI } \\
\hline Mean \pm SD & $28.85 \pm 4.23$ & $28.75 \pm 2.78$ & \multirow{2}{*}{0.888} \\
\hline Range & $21.79-40.23$ & $21.88-35.56$ & \\
\hline \multicolumn{4}{|c|}{ Systolic Blood Pressure } \\
\hline Mean \pm SD & $117.8 \pm 7.57$ & $123.2 \pm 7.94$ & \multirow{2}{*}{$0.001^{* *}$} \\
\hline Range & $100-130$ & $110-140$ & \\
\hline \multicolumn{4}{|c|}{ Diastolic Blood Pressure } \\
\hline Mean \pm SD & $78.7 \pm 7.61$ & $80.7 \pm 6.93$ & \multirow{2}{*}{0.173} \\
\hline Range & $65-90$ & $70-90$ & \\
\hline
\end{tabular}

Table 5: laboratory investigations of diabetic nephropathy patients according to grade of nephropathy.

\begin{tabular}{|c|c|c|c|c|}
\hline \multicolumn{2}{|c|}{ Grade 1} & Grade 2 & Grade 3 & $\mathbf{P}$ \\
\hline \multicolumn{5}{|c|}{ Vitamin D } \\
\hline Mean \pm SD & $4.49 \pm 0.33$ & $3.22 \pm 0.43$ & $2.19 \pm 0.27$ & \multirow{2}{*}{$<0.001^{* *}$} \\
\hline Range & $4.1-5$ & $2.6-4$ & $1.7-2.5$ & \\
\hline \multicolumn{5}{|c|}{ Serum Creatinine } \\
\hline Mean \pm SD & $1.05 \pm 0.19$ & $1.33 \pm 0.24$ & $2.02 \pm 0.32$ & \multirow{2}{*}{$<0.001^{* *}$} \\
\hline Range & $0.8-1.4$ & $1-2$ & $1.5-2.5$ & \\
\hline \multicolumn{5}{|l|}{ Albumin } \\
\hline Mean \pm SD & $4.1 \pm 0.31$ & $3.9 \pm 0.27$ & $3.34 \pm 0.31$ & \multirow{2}{*}{$<0.001^{* *}$} \\
\hline Range & $3.7-4.8$ & $3.5-4.5$ & $2.8-4$ & \\
\hline \multicolumn{5}{|c|}{ eGFR } \\
\hline Mean \pm SD & $105.33 \pm 7.26$ & $80.81 \pm 5.98$ & $51.97 \pm 6.01$ & \multirow{2}{*}{$<0.001^{* *}$} \\
\hline Range & $92.47-116.66$ & $68.3-88.4$ & $41.05-58.5$ & \\
\hline \multicolumn{5}{|c|}{ Albumin/Creatinine Ratio } \\
\hline Mean \pm SD & $93.03 \pm 41.88$ & $127.13 \pm 55.92$ & $574.43 \pm 379.56$ & \multirow{2}{*}{$<0.001^{* *}$} \\
\hline Range & $35.9-233.3$ & $54.8-231.4$ & $66.57-1227.3$ & \\
\hline \multicolumn{5}{|c|}{ HbAIc } \\
\hline Mean \pm SD & $9.19 \pm 1.21$ & $8.93 \pm 0.98$ & $9.34 \pm 1.28$ & \multirow{2}{*}{0.582} \\
\hline Range & $7.2-11.2$ & $7.2-11.3$ & $7.5-12.1$ & \\
\hline
\end{tabular}

Note:

a. Grade I nephropathy according to eGFR: more than 90.

b. $\quad$ Grade 2 nephropathy according to eGFR: 90-60.

c. Grade 3 nephropathy according to eGFR: 60-30. 
Table 6: Correlation between vitamin $\mathrm{D}$ and other variables.

\begin{tabular}{|c|c|c|c|c|c|c|}
\hline \multicolumn{3}{|c|}{ Control Group } & \multicolumn{2}{|c|}{ Diabetics without Nephropathy } & \multicolumn{2}{|c|}{ Diabetics with Nephropathy } \\
\hline \multirow{2}{*}{ Age } & $\mathrm{r} \infty$ & $\mathrm{p}$ & $r \infty$ & $\mathrm{p}$ & $\mathrm{r} \infty$ & $\mathrm{p}$ \\
\hline & -0.015 & 0.859 & 0.246 & 0.085 & -0.057 & 0.695 \\
\hline BMI & -0.158 & 0.273 & -0.213 & 0.138 & -0.07 & 0.63 \\
\hline $\begin{array}{l}\text { Systolic blood } \\
\text { pressure }\end{array}$ & 0.112 & 0.438 & 0.248 & 0.083 & -0.234 & 0.102 \\
\hline $\begin{array}{l}\text { Diastolic blood } \\
\text { pressure }\end{array}$ & 0.083 & 0.565 & 0.26 & 0.068 & -0.236 & 0.157 \\
\hline Serum creatinine & -0.415 & $0.003^{*}$ & -0.162 & 0.262 & -0.765 & $<0.001^{* *}$ \\
\hline Serum albumin & 0.05 & 0.728 & 0.584 & $<0.001^{* *}$ & 0.65 & $<0.001^{* *}$ \\
\hline eGFR & 0.331 & $0.019^{*}$ & 0.039 & 0.789 & 0.884 & $<0.001^{* *}$ \\
\hline \multicolumn{2}{|c|}{ Albumin/creatinine ratio } & & -0.061 & 0.675 & -0.64 & $<0.001^{* *}$ \\
\hline HbAIc & & & -0.807 & $<0.001^{* *}$ & 0.005 & 0.974 \\
\hline
\end{tabular}

\section{Discussion}

Vitamin D status can show associations with diabetic complications including nephropathy, neuropathy and retinopathy [10]. Vitamin D plays an important role in diabetic kidney disease through several pathways such as reducing oxidative stress and inflammation, maintaining podocyte healthy in the kidneys, suppressing renin gene transcription [11]. Our study revealed that in diabetic group, serum creatinine was higher, however serum albumin, eGFR were lower than control group. Vitamin D serum level tended to be lower in diabetic patients as shown in Table 2. This was in agreement with Bener, et al. [12]; who studied 170 diabetic patients and 170 control subjects and showed that vitamin D deficiency was considerably higher in diabetic patients $(90.6 \%)$ compared to non-diabetic population (85.3\%).Our study revealed that the systolic blood pressure was higher in diabetic nephropathy group than non-nephropathy group as shown in Table 3. Marsida, et al. [13]; agreed with this study that higher blood pressures are associated with diabetic nephropathy; his study was conducted on 900 diabetic patients to define risk factors for diabetic complications.

Our study showed that vitamin D levels was lower in diabetic group with nephropathy than those without it and moreover its level was lower in grade 3 nephropathy than grade $1 \& 2$ (Tables 4 $\& 5)$. Xiao, et al. [14] study was done on 240 diabetic nephropathy patients and 60 healthy control subjects found that $25(\mathrm{OH})$ vitamin D levels were lower in diabetic nephropathy patients than in controls and showed a gradually decreasing trend with diabetic nephropathy stage. Yuste, et al. [15] agreed also with our results; and found that 25-(OH) vitamin D3 deficiency had a high prevalence in CKD patients, and the severity increases with the progression of kidney disease. Also, Serum creatinine, albumin /creatinine ratio and $\mathrm{HbA1c}$ were higher in diabetic group with nephropathy than those without. However, eGFR was lower in diabetic group with nephropathy (Table 4). Fan, et al. [16]; studied 287 diabetic patients and were categorized in diabetic nephropathy group
(148 cases) and non-diabetic nephropathy group(139 cases) and found that Vitamin D deficiency is closely correlated with diabetic nephropathy and can be considered as a risk factor for diabetic nephropathy.

Derakhshanian, et al. [17]; meta-analysis showed a significant inverse association between serum vitamin D status and risk for nephropathy in patients with diabetes. However, no significant change following vitamin D supplementation was found alter neither the disease progression nor support causality. In our study HbA1c was higher in diabetic nephropathy patients than diabetic patients without nephropathy (Table 4). However, there was no significant difference regarding it between grades of nephropathy (Table 5). Serum creatinine and albumin \creatinine ratio were higher in grade 3 nephropathy than other groups. However, serum albumin and eGFR were lower in grade 3 nephropathy compared to other groups (Table 5). Sánchez-Hernández, et al. [18]; study was done on 113 patients with diabetes related CKD, 61 patients with long lasting type 2 diabetes without advanced CKD and 80 normal subjects without diabetes showed that low vitamin D status was associated with advanced diabetic nephropathy. This relationship was not entirely attributable to the individual effects of CKD or long-lasting diabetes.

However, Subhashini, et al. [19]; collected data from 27098 diabetic patients and found that there was no significant difference in $25(\mathrm{OH})$ vitamin D levels between subjects with diabetes and glomerular filtration rate (e-GFR) $<60$ compared to those with e-GFR $\geq 60$.The explanation the progressive decrease in vitamin D level with the different stages of CKD may be due to the progressive loss of renal 1-hydroxylase with the reductions in functional renal mass, the inhibition of remnant 1-hydroxylase activity by elevations in serum levels of uremic toxins and increase megalin expression (a multiligand receptor mediates the active process of endocytosis of the filtered 25 vitamin D bound to its carrier, the vitamin D-binding protein, from the glomerular ultra-filtrate). Serum creatinine and GFR decline in the natural course of CKD 
progression, while the serum albumin decline may be due to proteinuria and progressive loss of renal tissue while albumin/ creatinine ratio increase due to progressive increase of urinary protein excretion. In diabetic patients without nephropathy, there were significant negative correlation between vitamin D and HbAIc and positive correlation with serum albumin. Whereas in diabetic patients with nephropathy, vitamin D serum level had significant negative correlations with serum creatinine and albumin/ creatinine ratio, but it had significant positive correlation with serum albumin and eGFR (Table 6). Kajbaf, et al. [20]; studied 245 diabetic patients with nephropathy in different stages of CKD and found that $25(\mathrm{OH})$ vitamin D levels were negatively correlated with HbAIc levels. Lim, et al. [21]; also agreed with our results, his study was conducted on 100 type 2 diabetes patients with stage 3-4 CKD and found Lower serum $25(\mathrm{OH})$ vitamin D was associated with poorer glycemic control and higher insulin use among multi-ethnic Asians with type 2 diabetes stage 3-4 CKD.

\section{Conclusion}

Vitamin D is deficient in diabetic patients; it appears to be lower in diabetic patients with nephropathy and tends to be lower with progression of CKD stage in diabetic patients with nephropathy. We recommend further studies to show whether supplementation of vitamin D to diabetic patients will alter pathologic processes related to diabetic nephropathy.

\section{Disclosures/Conflict of interest}

The authors declare that there is no conflict of interest.

\section{References}

1. Galtier F (2010) Definition, epidemiology, risk factors. Diabetes Metab 36: 628-651.

2. Jorge L Gross, Mirela J de Azevedo, Canani LH, Caramori ML, Zelmanovitz T (2005) Diabetic nephropathy: diagnosis, prevention, and treatment. Diabetes Care 28(1): 164-176.

3. Fineberg Daniel, Karin AM, Jandeleit Dahm (2013) Diabetic Nephropathy: Diagnosis and Treatment. Nature Reviews. Endocrinology 9(12): 713-723.

4. Michael F, Holick (2011) Vitamin D: A lightful solution for health. J Investig Med 59(6): 872-880.

5. Alshahrani FM, AlmalkiMH, Aljohani N, Alzahrani A, Alsaleh Y, et al (2013) Vitamin D: Light side and best time of sunshine in Riyadh, Saudi Arabia. Dermatoendocrinol 5: 177-180.

6. Zhuo L, Ren W, Li W, Zou G, Lu J (2012) Evaluation of Renal Biopsies in Type 2 Diabetic Patients with Kidney Disease: A Clinicopathological Study of 2I6 Cases. International Urology and Nephrology 45(1).
7. (2015) American diabetes Association: Classification and Diagnosis of Diabetes; Diabetes Care 38(Supplement 1): S8-S16.

8. (2018) American Diabetes Association: Microvascular Complications and Foot Care: Standards of Medical Care in Diabetes-20I8 Diabetes Care 41(Supplement 1): S105-S118.

9. Cockcroft DW, MH Gault (1976) Prediction of creatinine clearance from serum creatinine. Nephron 16(1): 31-41.

10. Zhukouskaya VV, Eller-Vainicher C, Shepelkevich AP, Dydyshko Y, Cairoli E, et al. (2015) Bone health in type I diabetes: focus on evaluation and treatment in clinical practice. J Endocrinol Invest 38: 941-950.

11. Yuan W, Pan W, Kong J, Zheng W, Szeto FL, et al. (2007) I,25-dihydroxyvitamin D3 suppresses renin gene transcription by blocking the activity of the cyclic AMP response element in the renin gene promoter. J Biol Chem 282: 2982I-29830.

12. Bener A, Zirie M, Musallam M, Khader YS, Al-Hamaq AO (2009) Prevalence of metabolic syndrome according to Adult Treatment Panel III and International Diabetes Federation criteria: A population-based study. Metab Syndr Relat Disord 7: 22I-229.

13. Teliti M, Cogni G, Sacchi L, Dagliati A, Marini S, et al. (2018) Risk factors for the development of micro-vascular complications of type 2 diabetes in a single-centre cohort of patients. Diab Vasc Dis Research 5(5): 424432.

14. Xiaoyan Xiao,Yajuan Wang, Yanlian Hou, Feng Han, Jianmin Ren, et al. (2016) Vitamin D deficiency and related risk factors in patients with diabetic nephropathy.J Int Med Res 44(3): 673-684.

15. Fernández-Juárez G, Luño J, Barrio V, de Vinuesa SG, Praga M, et al. (2013) 25 (OH) Vitamin D Levels and Renal Disease Progression in Patients with Type 2 Diabetic Nephropathy and Blockade of the Renin-Angiotensin System. Clin J Am Soc Nehp 8(11): 1870-1876.

16. Fan L, Zhang Y, Zhu J, Song Y, Lin J (2018) Association of vitamin D deficiency with diabetic peripheral neuropathy and diabetic nephropathy in Tianjin, China.Asia Pac J Clin Nutr 27(3): 599-606.

17. Derakhshanian H, Shab-Bidar S, Speakman JR, Nadimi H, Djafarian K (2015) Vitamin D and diabetic nephropathy: A systematic review and meta-analysis. Nutrition 31(10): 1189-1194.

18. Sanchez-Hernandez, Garcia-Canton, Lorenzo, Quevedo V, Bosch E, et al. (2015) The specific relationship between vitamin D deficiency and diabetic nephropathy among patients with advanced chronic kidney disease: a cross-sectional study in Gran Canaria, Spain. Clin Nephrol 83(4): 218-224.

19. Subhashini Y, Barbara Y, Sonya Z (2017) Vitamin D levels in subjects with or without chronic kidney disease among Veterans with diabetes in North East United States. World J Diabetes 8(7): 346-350.

20. Kajbaf F, Mentaverri R, Diouf M, Fournier A, Kamel S, et al. (2014) The Association between 25-Hydroxyvitamin D and Hemoglobin A1c Levels in Patients with Type 2 Diabetes and Stage 1-5 Chronic Kidney Disease. Int J Endocrinol pp. 142468.

21. Lim LL, Ng YM, Kang PS, Lim SK (2018) Association between serum 25-hydroxyvitamin $\mathrm{D}$ and glycated hemoglobin levels in type 2 diabetes patients with chronic kidney disease. J Diabetes Investig 9(2): 375-382. 
ISSN: 2574-1241

DOI: 10.26717/BJSTR.2020.25.004237

Mohamed A Helaly. Biomed J Sci \& Tech Res

(C) $(9)$ This work is licensed under Creative

Submission Link: https://biomedres.us/submit-manuscript.php

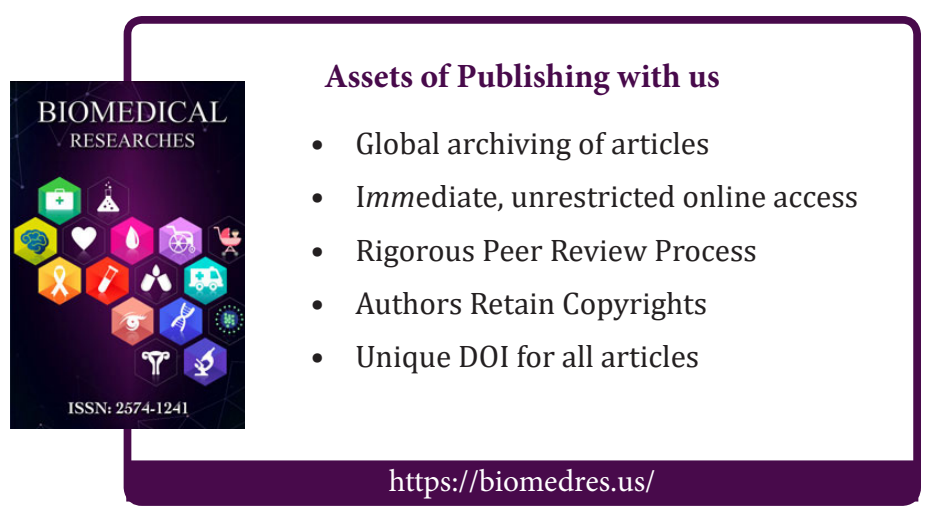

